\title{
Fit für die Zukunft: Vorschläge für eine moderne Sozialversicherung
}

Die Sozialversicherung als modern zu bezeichnen, mag Kenner der Debatten um die Reform des Sozialstaats überraschen. ${ }^{1}$ Verglichen mit der Gestaltung sozialer Sicherung in Form einer universellen Staatsbürgerversorgung (skandinavisches Modell) oder nachrangig gewährter Fürsorge (angelsächsisches Modell) stand das kontinentaleuropäische Sozialversicherungsmodell in den letzten Jahrzehnten besonders stark in der Kritik. Für die einen ist es wegen seiner Beitragsfinanzierung ein Standortnachteil in Zeiten der Globalisierung. Für andere verletzt es aufgrund der Lohnarbeitszentrierung und der damit verbundenen Statusfortschreibung grundlegende Aspekte sozialer Gerechtigkeit und gesellschaftlicher Umverteilung. Darüber hinaus sind "neue soziale Risiken“ nur unzureichend abgesichert. ${ }^{2}$ Die Systeme sozialer Sicherung in den Sozialversicherungsländern leiden zudem unter Finanzierungsproblemen, da Massenarbeitslosigkeit und Wachstumsschwäche die Finanzierungsbasis aushöhlen.

Das skandinavische Modell wurde von vielen Autoren dagegen als überlegen herausgestellt, da es ein hohes Maß an gesellschaftlicher Gleichheit mit wirtschaftlichem Erfolg vereint. Nicht ohne Grund hat Manow $^{3}$ zur Umschreibung der drei Wohlfahrtsstaatswelten nach Esping-Andersen die Begriffe „the good“ (Skandinavien), "the bad" (der liberale Wohlfahrtsstaat) und "the ugly" (die kontinentalen Sozialstaaten) geprägt. Der Begriff „ugly“ steht dafür, dass es sich aus Esping-Andersens Perspektive beim konservativen Wohlfahrtsstaat um ein ,unentschlossenes Regime" handle. Es stehe zwar für mehr Marktkorrekturen als das liberale Modell, gehe aber im egalitären Anspruch weniger weit als das universalistische sozialdemokratische Regime. Anders als das Letztgenannte erscheint der konservative Wohlfahrtsstaat als ein System mit begrenzter Umverteilung, hohem Statuserhalt, Paternalismus sowie Anreizen für eine patriarchale soziale Ordnung. Vielfach schweifte in den vergangenen Jahren daher der Blick gen Nor- den oder Westen auf den universellen oder den marktliberalen Wohlfahrtsstaat als Vorbild.

Entgegen dieser Ansicht stellen wir die Auffassung zur Diskussion, dass die Sozialversicherung zukunftsfähig ist. Sie ist zwar reformbedürftig (vgl. den ausführlichen Problemaufriss von Seils in diesem Heft zu den Themen Arbeitnehmerzentrierung, Transferlastigkeit und Finanzierung der Sozialversicherung), und die eingangs genannten Kritikpunkte sind nicht ohne Berechtigung. Doch bietet die Sozialversicherung, so unsere These, hinreichend Flexibilität, um den identifizierten Schwächen zu begegnen, ohne gleichzeitig ihre Stärken durch eine komplette Abkehr vom System preisgeben zu müssen.

\section{VERGESSENE STÄRKEN DER SOZIALVERSICHERUNG}

Die prinzipiellen Stärken der Sozialversicherung gerieten in den politischen Diskussionen der vergangenen Jahre oft in Vergessenheit, wenngleich die aktuelle Finanz- und Wirtschaftskrise einige davon wieder in Erinnerung ruft. Gerade in Krisenzeiten erfüllt die Sozialversicherung eine wichtige Funktion der makroökonomischen Stabilisierung (vgl. Meinhardt in diesem Heft). Und auch die finanzielle „Nachhaltigkeit“ von umlagefinanzierten gegenüber kapitalgedeckten Sicherungssystemen rückt wieder in ein realistischeres Licht.

Allein die Sozialversicherung ist zudem prinzipiell geeignet, soziale Sicherungsleistungen an das durchschnittliche Einkommensniveau einer Gesellschaft anzubinden, eigentumsrechtliche Anwartschaften zu begründen und im Bedarfsfall eine Sicherung des Lebensstandards zu gewährleisten. Deshalb genießt sie eine hohe soziale Akzeptanz. Ein weiterer wesentlicher Unterschied zu steuerfinanzierten Systemen der Fürsorge oder Staatsbürgerversorgung liegt in der Einbeziehung der Versicherungsnehmer in die Organisation und Verwaltung der Systeme. Die Sozialversi- cherung ist damit nicht allein dem Willen einer Regierung unterworfen, sondern die Hauptleistungsträger und (potenziellen) Leistungsempfänger sind in die Gestaltung einbezogen.

In der Sozialversicherung ist zudem ein sozialer Ausgleich angelegt, der versicherungsspezifische Risiken umverteilt (z. B. bringen in der Arbeitslosenversicherung diejenigen höhere Leistungen auf, deren Arbeitslosigkeitsrisiko niedriger ist). Ergänzend findet über die einkommensabhängige Beitragsgestaltung eine Umverteilung von Personen mit hohem Einkommen zu Personen mit niedrigem Einkommen statt. Dieser soziale Ausgleich ist jedoch in der Arbeitslosen- und Rentenversicherung begrenzt durch das Prinzip der Beitragsäquivalenz. Dieses bestimmt, dass die zu erwartenden Leistungen in Relation zur Höhe (in der Rentenversicherung auch zur

$1 \quad$ Wir danken ganz herzlich Silke Bothfeld und Eric Seils für anregende Diskussionen und Ideen zu diesem Thema im Kontext unserer gemeinsamen Arbeit am WSI-Projekt "Sozialversicherung als Zukunftsmodell: deutsche und internationale Perspektiven“, http://www.boeckler.de/projektlist_ projekte_wsi_91895.html.

2 Darunter versteht man in der Regel Risiken, die durch die Transformation zur postindustriellen Gesellschaft entstanden sind, z. B. Taylor-Gooby, P. (2004): New Risks, New Welfare: The Transformation of the European Welfare State, Oxford, S. $2 f$.

3 Manow, P. (2002): 'The Good, the Bad, and the Ugly'. Esping-Andersens Wohlfahrtsstaatstypologie und die konfessionellen Grundlagen des westlichen Sozialstaats, in: Kölner Zeitschrift für Soziologie und Sozialpsychologie 2, S. 203-225.

Claudia Bogedan, Wissenschaftlerin im WSI in der Hans-Böckler-Stiftung, Arbeitsschwerpunkte: Arbeitsmarktpolitik, vergleichende Wohlfahrtsstaatsforschung.

e-mail: claudia-bogedan@boeckler.de Simone Leiber, Dr., Wissenschaftlerin im WSI in der Hans-Böckler-Stiftung. Arbeitsschwerpunkte: Wohlfahrtsstaaten im Vergleich, Europäische Sozialpolitik, Gesundheitspolitik.

e-mail: simone-leiber@boeckler.de 
Dauer) der geleisteten Beiträge stehen. Die Sozialversicherung wirkt somit in einigen Zweigen statuskonservierend. Dies kann dann zum Problem werden, wenn unterschiedliche Gesellschaftsgruppen systematisch keinen Zugang zu einer hinreichenden Versorgung über diese Sicherungsinstitutionen haben (Seils in diesem Heft). Grundsätzlich garantiert die Sicherung der relativen sozialen Stellung aber die Unterstützung der Mittelschichten, da sie einen sozialen Abstieg im Risikofall verhindert und eine "Gegenleistung " für gezahlte Beiträge gesichert ist. Dies stellt einen grundlegenden Unterschied zu steuerfinanzierten Systemen dar, da Steuern nicht zweckgebunden erhoben werden. ${ }^{4}$

Anstelle eines (schleichenden) Umstiegs und der Preisgabe der Sozialversicherung in ihrem Kern sind folglich Reformalternativen zu präferieren, die Sozialversicherungselemente auf kluge Weise mit Wettbewerbs- oder universalistischen Elementen verbinden. Das innovative Potenzial kombinierter Lösungen auszuloten und ihre Übertragbarkeit auf die deutschen Sicherungssysteme zu prüfen, ist aus unserer Sicht eine zentrale Herausforderung der Zukunft, für die in diesem Heft erste Diskussionsstränge entwickelt werden.

\section{SCHRITTE AUF DEM WEG ZUR MODERNEN SOZIALVERSICHERUNG}

Eine solche Weiterentwicklung der Sozialversicherung, so die These, ermöglicht es, den politischen Willen vorausgesetzt, ohne die hohen Transaktionskosten eines Systemwechsels die bestehenden Sicherungsprobleme zu beheben. Dazu gehören die Universalisierung und Vereinheitlichung des Zugangs, der Ausbau mindestsichernder Elemente, die Stärkung der Dienstleistungsdimension und präventiver Maßnahmen sowie die Erweiterung der Finanzierungsbasis. Die Beiträge des Heftes geben vor dem Hintergrund internationaler Erfahrungen Hinweise, wie diese Ziele künftig erreicht werden könnten.
Problem Arbeitnehmerzentrierung: Um den Zugang zu Sicherungsleistungen zu vereinheitlichen, müssten einkommensund berufsbezogene Sondersysteme, wie die private Krankenvollversicherung, in den Rahmen des öffentlichen Versicherungssystems integriert werden. Nur so können ungleiche Wettbewerbs- und Versorgungsbedingungen ausgeglichen werden (Beitrag Greß et al.). Für den Bereich der Alterssicherung könnte der Ausbau der Gesetzlichen Rentenversicherung (GRV) zu einer Erwerbstätigenversicherung (Beiträge Thiede und Schmähl) - gegebenenfalls in Kombination mit weiteren mindestsichernden Elementen - die Armutsfestigkeit des Systems erhöhen und dazu beitragen, insbesondere Sicherungslücken von (Solo-)Selbstständigen zu schließen. Elemente der Mindestsicherung unterstützen auch die Absicherung unsteter Erwerbsverläufe (Beitrag Leschke). Ergänzend wären der Abbau von Zugangshürden atypisch Beschäftigter (vor allem Leiharbeiter und befristet Beschäftigte) zur betrieblichen Altersvorsorge (wie etwa Verbesserung der Portabilität und Anpassungsfähigkeit an Erwerbsunterbrechungen) sowie die Absenkung von Stunden- und Einkommensschwellen in der Arbeitslosenversicherung hilfreich. Um atypisch Beschäftigte nicht zu benachteiligen, müssten - anders als derzeit in Deutschland - individuelle Leistungen der Arbeitslosenversicherung unabhängig von der vorherigen Beitragszeit gewährt werden. Auch könnte eine Abgabe in Betracht gezogen werden, die Arbeitgeber, welche starken Gebrauch von befristeter Beschäftigung oder Zeitverträgen machen, finanziell an den erhöhten Ausgaben für Arbeitslosigkeit beteiligt.

Problem Transferlastigkeit: Der Ausbau sozialer Dienstleistungen und präventiver Maßnahmen sorgt zudem für eine bessere Absicherung „neuer sozialer Risiken“. Leitner (in diesem Heft) plädiert neben einem weiteren Ausbau der öffentlichen Betreuungsinfrastruktur in den Bereichen Kinderbetreuung und Pflege für eine indi- viduelle Existenzsicherung von Familienarbeitenden über das Eltern- bzw. Pflegegeld. Außerdem könnte die Weiterbildung, so Hipp (in diesem Heft), durch den Umbau der Arbeitslosen- zur Beschäftigungsversicherung systematisch gestärkt werden.

Problem Finanzierungsbasis: Die wirtschaftliche Tragfähigkeit einer solchen modernen Sozialversicherung fußt auf drei Säulen: Erstens wird durch die Universalisierung des Zugangs die Finanzierungsbasis verbreitert. Zweitens legitimiert das Konzept von Schmähl (in diesem Heft) der „aufgabenadäquaten Finanzierung" die Übernahme gesamtgesellschaftlich wünschenswerter (sogenannter versicherungsfremder) Leistungen in der Sozialversicherung. Dieses umfasst einen weiteren Ausbau der Steuerfinanzierung. Für die GRV schlägt Schmähl vor, dass der Staat seine Beitragsleistungen zur Rentenversicherung für Arbeitslose, Auszubildende und Personen, die Pflege- und Betreuungstätigkeiten ausüben, weiter erhöht und Leistungen für Hinterbliebene aus Steuermitteln finanziert werden. Drittens zeigt Meinhardt (in diesem Heft), dass ein ausgebauter Wohlfahrtsstaat, anders als in der liberalen Wirtschaftstheorie unterstellt, kein Wachstumshemmnis sein muss, sondern ihm im Gegenteil sogar eine besondere Bedeutung als Wirtschaftsmotor zukommt.

Die Beiträge untermauern die These: Die Weiterentwicklung der Sozialversicherung in Richtung eines tragfähigen und modernen Sicherungssystems ist nicht prinzipiell verstellt. Es ist vielmehr eine Frage der politischen Kräfteverhältnisse, ob und wie alte und neue soziale Risiken künftig sozialstaatlich abgesichert werden sollen.

4 Dies war unter anderem ein Grund, warum skandinavische Länder sukzessive Sozialversicherungselemente in ihre Sicherungssysteme integriert haben. 